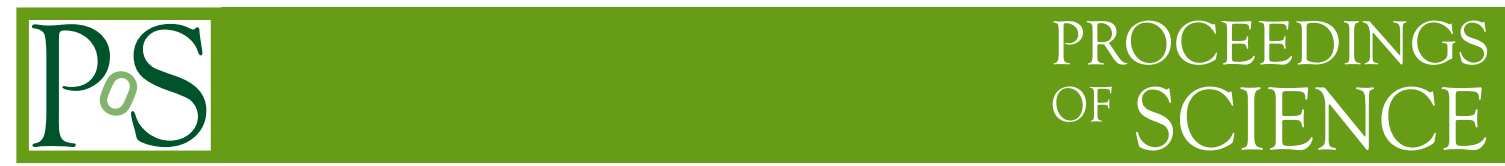

\title{
2-forms in holographic QCD
}

\author{
Oscar Catà *中 \\ Departament de Física Teòrica, IFIC, Universitat de València, Apt. Correus 22085, E-46071 \\ València, Spain, \\ E-mail: oscar.cataeific.uv.es
}

A complete description of spin-1 states in holographic models of QCD requires to take into consideration not only 1 -forms but also 2 -forms on the gravitational side. I show how the nontrivial entwinement between them in the description of vector mesons leads to a very constrained picture in which, for instance, chiral symmetry breaking emerges naturally. Some phenomenological applications are also discussed.

Light Cone 2010 - LC2010

June 14-18, 2010

Valencia, Spain

\footnotetext{
* Speaker.

${ }^{\dagger}$ Work supported in part by the EU under contract MTRN-CT-2006-035482 (FLAVIAnet) and by MICINN, Spain under the Juan de la Cierva program and grants FPA2007-60323 and Consolider-Ingenio 2010 CSD2007-00042 (CPAN).
} 


\section{Introduction}

The AdS/CFT correspondence [1] currently stands out as a promising tool to gain insight into the nonperturbative regime of QCD. The correspondence conjectures the existence of 5-dimensional Anti-de Sitter gravitational theories whose weakly coupled limit is dual to the strongly coupled regime of 4-dimensional (conformal) gauge theories. Obviously there is a big leap from the original conjecture to the statement that QCD may have a gravitational dual: firstly, while QCD at high (Euclidean) energies is certainly conformal, this corresponds to its weakly coupled regime; and secondly, the complexity of the QCD vacuum requires to include confinement and chiral symmetry breaking in the picture. Even so, there is increasing evidence that very simple gravitational actions are already able to capture the most relevant features of QCD.

This phenomenologically oriented approach usually relies on simple geometric configurations like the one depicted in Figure 1, where the bulk 5-dimensional AdS space is limited by 4-dimensional boundary branes: the left-handed UV brane is where the gauge theory lives, while the right-handed one (the IR brane) mimicks all the genuine nonperturbative physics: it breaks conformal symmetry, sets a confinement scale, triggers spontaneous chiral symmetry breaking and generates a hadronic spectrum. Phenomenological studies with such configurations have found remarkable agreement with experimental results, especially for spin-1 particles created by the vector $J_{\mu}=\bar{q} \gamma_{\mu} q$ and axial $J_{\mu}^{5}=\bar{q} \gamma_{\mu} \gamma^{5} q$ currents [2].

However, the picture for spin-1 particles is not so simple in QCD. It is well known that vector mesons can also be created by the tensor current $J_{\mu \nu}=\bar{q} \sigma_{\mu \nu} q$, such that

$$
\begin{aligned}
\left\langle 0\left|J_{\mu}\right| \rho_{n}(p, \lambda)\right\rangle & =f_{V n} m_{V n} \varepsilon_{\mu}^{(\lambda)} \\
\left\langle 0\left|J_{\mu v}\right| \rho_{n}(p, \lambda)\right\rangle & =i f_{V n}^{\perp}\left(\varepsilon_{\mu}^{(\lambda)} p_{v}-\varepsilon_{v}^{(\lambda)} p_{\mu}\right) .
\end{aligned}
$$

Recall that the holographic recipe associates 5-dimensional fields with 4-dimensional currents, so it is far from clear how the duplicity shown in the previous equation can be accomodated in the holographic picture. Furthermore, as soon as $J_{\mu v}$ is considered, one also generates (even-parity) spin-1 states

$$
\left\langle 0\left|J_{\mu v}\right| b_{n}(p, \lambda)\right\rangle=i f_{B n} \varepsilon_{\mu \nu \eta \rho} \varepsilon_{(\lambda)}^{\eta} p^{\rho} .
$$

Therefore, in order to complete the picture for spin-1 mesons, not only vector but also tensor currents have to be taken into account. Following the holographic recipe, this corresponds to including 2-forms on the gravity side. However, the fact that $1^{--}$is sensitive to 1 -form and 2forms, and that both $1^{--}$and $1^{+-}$states are created by $J_{\mu \nu}$ implies that strong correlations are to be expected. This can be seen in the following set of 2-point Green's functions, which in the large- $N_{c}$ limit can be expressed as

$$
\begin{aligned}
& \Pi_{V V}\left(q^{2}\right)=\sum_{n}^{\infty} \frac{f_{V n}^{2}}{-q^{2}+m_{V n}^{2}}, \\
& \Pi_{T T}^{+}\left(q^{2}\right)=\sum_{n}^{\infty} \frac{f_{B n}^{2}}{-q^{2}+m_{B n}^{2}}, \\
& \Pi_{T T}^{-}\left(q^{2}\right)=\sum_{n}^{\infty} \xi_{n}^{2} \frac{f_{V n}^{2}}{-q^{2}+m_{V n}^{2}}
\end{aligned}
$$



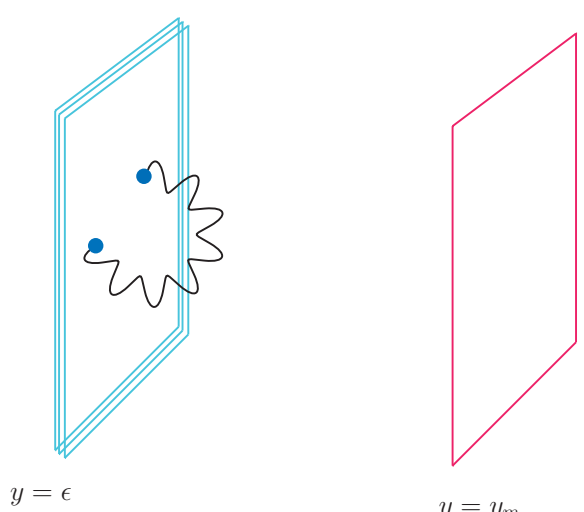

$y=y_{m}$

Figure 1: Geometric setting for hard-wall holographic models of QCD.

$$
\Pi_{V T}\left(q^{2}\right)=\sum_{n}^{\infty} \xi_{n} \frac{f_{V n}^{2} m_{V n}}{-q^{2}+m_{V n}^{2}},
$$

where $\xi_{n} \equiv f_{V n}^{\perp} / f_{V n}$. The simultaneous analysis of the previous correlators leads to a series of bootstrapping relations $[3,4]$ which will strongly constraint the resulting phenomenology.

\section{A minimal action for 2 -forms}

As discussed in the previous section, we will consider a 5-dimensional action for both 1-forms and 2-forms living in the configuration of Figure 1. For simplicity, we will consider the action

$$
S=\sum_{p=1,2} \kappa_{i} \int_{A d S_{5}} e^{-\Phi(y)} \operatorname{Tr}\left[d W_{p} \wedge^{*} d W_{p}+m_{p}^{2} W_{p} \wedge^{*} W_{p}\right]
$$

with the generic metric

$$
d s^{2}=g_{M N} d x^{M} d x^{N}=e^{2 A(y)}\left(-d y^{2}+\eta_{\mu \nu} d x^{\mu} d x^{v}\right), \quad \varepsilon<y \leq y_{m} .
$$

The 5-dimensional masses are given by the AdS/CFT recipe $m_{p}^{2}=(\Delta-p)(\Delta+p-d)$ and $\Phi(y)$ is a dilaton field. For the time being we will let the masses unspecified and set a trivial $\Phi(y)=\phi$ dilaton background. In components, the previous action then reads

$$
\begin{aligned}
& S_{1}=\lambda \int d^{5} x \sqrt{g} \operatorname{Tr}\left[-\frac{1}{2} F_{M N} F^{M N}+m^{2} V_{N} V^{N}\right], \\
& S_{2}=2 \kappa \int d^{5} x \sqrt{g} \operatorname{Tr}\left[-\frac{1}{2} \partial_{L} H_{M N} \partial^{M} H^{L N}+\frac{1}{4} \partial_{L} H_{M N} \partial^{L} H^{M N}-\frac{m^{2}}{4} H_{M N} H^{M N}\right] .
\end{aligned}
$$

Notice that we only consider the kinetic terms for each form and omit any interactions. This might seem surprising, given that $\Pi_{V T}$ seems to call for such an interaction. However, we will see later on that $\Pi_{V T}$ can be nonzero without interaction terms.

Following the holographic recipe, one would decompose the 5-dimensional fields in KaluzaKlein modes, such that $V_{N}=\left(V_{5}, V_{\mu}\right)$ and $H_{M N}=\left(H_{\mu 5}, H_{\mu v}\right)$ and solve for the equations of motion. 
The solutions, which can be expressed in terms of Bessel functions [5], are then to be plugged back into the original action. This way one ends up with a pure boundary action, whose expression when $A(y)=\log y$, i.e., in pure AdS metric, is given by the following expression: ${ }^{1}$

$$
S=\left.\int d^{4} x\left[\frac{\lambda}{2}\left\{\frac{1}{y} V^{\mu(a)} \partial_{y} V_{\mu}^{(a)}\right\}-\frac{\kappa}{4}\left\{y H^{\mu v(a)}\left(\partial_{y} H_{\mu \nu}^{(a)}-\partial_{\mu} H_{5 v}^{(a)}+\partial_{v} H_{5 \mu}^{(a)}\right)\right\}\right]\right|_{\varepsilon} ^{y_{0}},
$$

The on-shell 5-dimensional fields, projected on the UV brane, are to be identified with the sources of the gauge theory [6]. The previous action therefore allows us to compute 2-point correlators.

\section{Transversely polarized mesons from 5-dimensions}

A closer look at the previous action reveals that before actually computing any correlator several issues have to be clarified. First, while it is natural to identify $V_{\mu}(\varepsilon)$ and $H_{\mu v}(\varepsilon)$ as the sources of the $J_{\mu}$ and $J_{\mu \nu}$ currents, respectively, the interpretation of $V_{5}$ and $H_{\mu 5}$ fields is far from clear: these by-products of the Kaluza-Klein reduction do not seem to correspond to any QCD current. Furthermore, there is the unresolved issue that different currents $J_{\mu \nu}$ and $J_{\mu}$ can create the same hadronic $1^{--}$states, which seems to clash with the holographic recipe. And finally, it seems puzzling that one can generate mixed correlators like $\Pi_{V T}$ without mixing forms in the original 5-dimensional action.

In the following I will show that all these issues get resolved if $V_{5}$ and $H_{\mu 5}$ are interpreted as scalar and vector sources, respectively. To support this interpretation it is convenient to look at the equations of motion for $H_{\mu 5}$ and $V_{\mu}:^{2}$

$$
\begin{aligned}
\left(\partial_{y}^{2}-\frac{1}{y} \partial_{y}-\square+\frac{1-m_{H}^{2}}{y^{2}}\right) H_{5 \mu} & =0, \\
\left(\partial_{y}^{2}-\frac{1}{y} \partial_{y}-\square\right) V_{\mu} & =0,
\end{aligned}
$$

and notice that indeed they are equivalent only if $m_{H}=1$, which is precisely the mass assignment predicted by the holographic recipe. This is not an accident but rather a general feature of $p$-forms in holographic settings. Consider for instance the equations of motion for $V_{5}$ and a scalar field $\phi$ :

$$
\begin{aligned}
\left(\partial_{y}^{2}-\frac{3}{y} \partial_{y}-\square+\frac{3+m_{V}^{2}}{y^{2}}\right) V_{5} & =0, \\
\left(\partial_{y}^{2}-\frac{3}{y} \partial_{y}-\square+\frac{3}{y^{2}}\right) \phi & =0,
\end{aligned}
$$

which again are equivalent provided $m_{V}=0$, again the value predicted by holography.

Based on the previous equations and according to Eq. (2.4), one is naturally led to identify $H_{\mu 5}$ as the vector source that generates $\Pi_{V T}$ and $V_{5}$ as the scalar source in $\Pi_{S V}$. This interpretation can be shown to be consistent and provides natural explanations for observed patterns in QCD. For

\footnotetext{
${ }^{1}$ I include only terms quadratic in the fields, which are the relevant ones to compute 2-point functions at tree level.

${ }^{2}$ If one works out the equations of motion for 2-forms, $H_{\mu v}$ and $H_{\mu 5}$ are coupled. The decoupled expression for $H_{\mu 5}$ is found only after using the consistency condition $d^{*} H=0$, which is satisfied by any massive $p$-form.
} 
instance, experimentally one finds that $\Pi_{S V}=0$, which at least in the chiral and large- $N_{c}$ limits can be justified. According to the AdS/CFT correspondence, the fact that $m_{V}=0$ (which is linked to vector current conservation) means that $V_{N}$ is a 5-dimensional gauge field. Therefore, $V_{5}$ can actually be gauged away and indeed $\Pi_{S V}=0$. Likewise, the fact that $\Pi_{V T} \neq 0$ in QCD can be understood holographically as due to the non-conservation of the tensor current, in which case $H_{\mu 5}$ is no longer a gauge artifact but rather a physical field.

\section{A comment on dilaton backgrounds}

The interpretation of the residual $V_{5}$ and $H_{\mu 5}$ as scalar and vector fields in the previous Section might seem trivial just by looking at their Lorentz indices. This is however not so straightforward and the interpretation turns out to be a unique feature of pure AdS spaces without dilaton profiles.

The introduction of a dilaton field was motivated in Ref. [7] to force the spectrum of $1^{--}$ mesons to display Regge trajectories, which can be achieved by choosing $\Phi(y)=c y^{2}$. If this were a valid mechanism to incorporate linear confinement in the vector spectrum, it should also apply to $H_{\mu 5}$. However, what one observes from $H_{\mu 5}$ is that the dilaton field factors out from its equation of motion:

$$
\left(\square+m^{2} e^{2 A}\right) H_{5 v}-\partial_{v} \partial_{\alpha} H_{5 \alpha}-\partial_{y} \partial^{\alpha} H_{\alpha v}=0 .
$$

As mentioned before, this equation couples $H_{\mu 5}$ and $H_{\mu v}$, and it is convenient to use the condition $d^{*} H=0$, which in components reads

$$
\begin{aligned}
\partial^{\mu} H_{\mu 5} & =0, \\
\partial^{\mu} H_{\mu v} & =e^{-A} \partial_{y}\left(e^{A} H_{5 v}\right),
\end{aligned}
$$

to decouple $H_{\mu \nu}$. Notice however that the consistency condition does not introduce any dilaton dependence because it only depends on the metric. Therefore, the presence of a dilaton field affects $V_{\mu}$ but leaves $H_{\mu 5}$ untouched. As a result, if $V_{\mu}$ and $H_{\mu 5}$ are to be identified as the transverse and longitudinal sources for vector mesons, the presence of a dilaton is definitely ruled out. In particular, this implies that models which display linear confinement through dilatonic backgrounds do not provide a consistent description of vector mesons.

Finally, let me remark that the equation of motion for $H_{\mu v}$ does depend on the dilaton field, but the choice $\Phi(y)=c y^{2}$ does not lead to a spectrum with linear scaling.

\section{Bootstrapping the infrared: implications for chiral symmetry breaking}

In order to compute correlators the boundary conditions for the different fields have to be specified. Conditions on the UV brane are dictated by the holographic recipe and define the 4dimensional sources. In contrast, conditions on the IR brane determine the nonperturbative aspects of the theory and in principle there is a certain degree of freedom in choosing them.

For the vector field $V_{\mu}$ it is common to choose Neuman boundary conditions

$$
\partial_{y} \hat{V}\left(q, y_{m}\right)=0
$$


in order to prevent a zero mode in the vector channel. The point to stress here is that, once the IR boundary condition for $V_{\mu}$ is specified, it automatically determines the boundary conditions for $H_{\mu 5}$ and $H_{\mu \nu}$ to be

$$
\begin{aligned}
\partial_{y} \hat{H}\left(q, y_{m}\right) & =0, \\
\bar{H}\left(q, y_{m}\right) & =\rho(q) .
\end{aligned}
$$

The last condition turns out to be the only possibility to ensure single poles in $\Pi_{V T}$, where $\rho(q)$ is a function whose behavior can be inferred from long and short distance QCD constraints. Regardless of the specific form $\rho(q)$ takes, the Dirichlet condition ensures that $\Pi_{V T}$ has the same poles as $\Pi_{V V}$, in compliance with Eqs. (1.3). The first condition follows from the fact that if $H_{\mu 5}$ and $V_{\mu}$ are to describe the same states, not only the equation of motion but also the boundary conditions should be the same.

With the boundary conditions above, one can compute the following correlators:

$$
\begin{aligned}
\Pi_{\mu v}^{V V}(q) & =i \int \mathrm{d}^{4} x e^{i q \cdot x}\left\langle 0\left|T\left\{V_{\mu}(x) V_{v}^{\dagger}(0)\right\}\right| 0\right\rangle=\left(q_{\mu} q_{v}-q^{2} g_{\mu v}\right) \Pi_{V V}\left(q^{2}\right), \\
\Pi_{\mu ; v \rho}^{V T}(q) & =i \int \mathrm{d}^{4} x e^{i q \cdot x}\left\langle 0\left|T\left\{V_{\mu}(x) J_{v \rho}^{\dagger}(0)\right\}\right| 0\right\rangle=i\left(q_{\rho} g_{\mu v}-q_{v} g_{\mu \rho}\right) \Pi_{V T}\left(q^{2}\right), \\
\Pi_{\mu v ; \alpha \beta}^{T T}(q) & =i \int \mathrm{d}^{4} x e^{i q \cdot x}\left\langle 0\left|T\left\{J_{\mu v}(x) J_{\alpha \beta}^{\dagger}(0)\right\}\right| 0\right\rangle=\Pi_{T T}^{-}\left(q^{2}\right) F_{-}^{\mu v ; \alpha \beta}(q)+\Pi_{T T}^{+}\left(q^{2}\right) F_{+}^{\mu v ; \alpha \beta}(q),
\end{aligned}
$$

by functionally differentiating the action of Eq. (2.4). The results for the form factors are

$$
\begin{aligned}
\Pi_{V V}\left(q^{2}\right) & =-\lambda\left[\log \frac{q^{2}}{\mu^{2}}-\pi \frac{Y_{0}(\zeta)}{J_{0}(\zeta)}\right] \\
\Pi_{V T}\left(q^{2}\right) & =\frac{\kappa \pi}{4} y_{m}^{2} \rho(q)\left[Y_{1}(\zeta)-\frac{J_{1}(\zeta) Y_{0}(\zeta)}{J_{0}(\zeta)}\right], \\
\Pi_{T T}^{+}-\Pi_{T T}^{-} \equiv \Pi_{T T}^{ \pm}\left(q^{2}\right) & =\frac{\kappa}{4 q^{2}}\left[2+\zeta \rho^{2}(q) \frac{J_{2}(\zeta)-J_{0}(\zeta)}{J_{1}(\zeta)}\right] .
\end{aligned}
$$

The first thing to notice is the spectrum pattern that emerges. As anticipated, the boundary conditions guarantee that $\Pi_{V V}$ and $\Pi_{V T}$ both exchange $1^{--}$mesons, as they should, and their masses turn out to be located at the zeros of $J_{0}$. The situation with $\Pi_{T T}$ is a bit more involved. The structure of the action (2.4) provides direct access to $\Pi_{T T}^{ \pm}$, and from there one can reconstruct $\Pi_{T T}^{-}$and $\Pi_{T T}^{+}$. Notice that the former can be fully reconstructed from $\Pi_{V V}$ and $\Pi_{V T}$ alone, and therefore $\Pi_{T T}^{+}$can also be determined as $\Pi_{T T}^{+}=\Pi_{T T}^{ \pm}+\Pi_{T T}^{-}$. A schematic picture of the predicted spectrums for the different spin-1 states is shown in Figure 2. The striking feature is that the spectrum of $1^{+-}$states is predicted to be twice as dense, with half the states degenerate with vectors and half the states degenerate with axials. Although not much is known about these states beyond the first excitation $b_{1}(1235)$, it is tantalizing that $m_{b 1}=1229 \pm 3 \mathrm{MeV}$ while $m_{a 1}=1230 \pm 40 \mathrm{MeV}$.

Another interesting thing to point out is that the resonance structure of both $\Pi_{V T}$ and $\Pi_{T T}^{ \pm}$is proportional to $\rho(q)$. Since both correlators are order parameters of spontaneous chiral symmetry breaking, $\rho(q)$ is to be identified as the order parameter that triggers $\chi \mathrm{SB}$. Notice that $\chi \mathrm{SB}$ was 


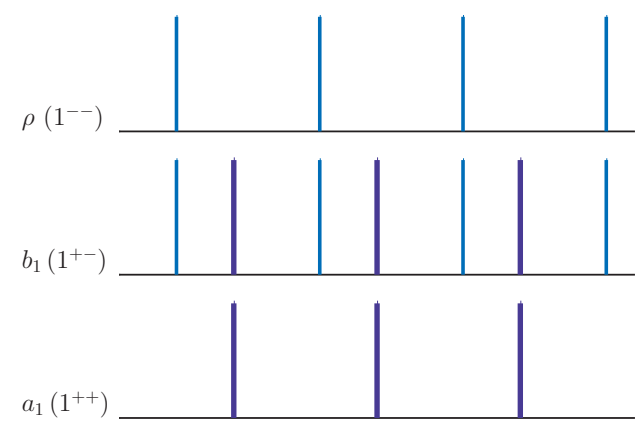

Figure 2: Spectrum of spin-1 particles predicted by the holographic model. $1^{--}$states lie at the zeros of $J_{0}$, while the masses for $1^{++}$axial states correspond to the zeros of $J_{1}$ [8].

never imposed in the model: it turned out to be induced by boundary conditions and it appeared naturally due to consistency conditions between the correlators. The boundary term $\rho(q)$ is obviously also responsible for the splitting between chiral partners depicted in Figure 2.

Notice that the effects of chiral symmetry breaking are indeed subtle in the correlators under study: nowhere a pion pole is allowed (by quantum numbers) to be exchanged, unlike in correlators like $\Pi_{A A}$ or $\Pi_{A P}$. The function $\rho(q)$ is certainly related to the quark condensate, but it would be interesting to show this relation in an explicit manner.

\section{Some phenomenological applications}

The correlators computed in Eq. (5.4) depend on a set of free parameters, namely $\lambda, \kappa$, the size of the fifth dimension $y_{m}$ and $\rho(q) . \lambda$ and $y_{m}$ can be fixed entirely from $\Pi_{V V}$, the former by matching the high energy behavior of $\Pi_{V V}$ to the QCD partonic logarithm, while $y_{m}$ is commonly chosen such that the first vector meson matches the $\rho$-meson mass. In contrast, $\kappa$ and $\rho(q)$ have to be determined by bootstrapping with the remaining correlators [5]. Once the parameters are fixed, one can study the ensuing phenomenology.

Here I will comment on two phenomenological examples, namely the $n$-dependence of the parameter $\xi_{n}$ and the magnetic susceptibility $\chi_{0}$.

The parameter $\xi_{\rho} \equiv f_{\rho}^{\perp} / f_{\rho}$ is a fundamental quantity in the evaluation of the CKM matrix element $\left|V_{u b}\right|$ and has been estimated by the lattice to be $\xi_{\rho}=0.72(2)$ [9]. In the large- $N_{c}$ limit, one can show that for large excitation numbers $\xi_{n} \sim(-1)^{n} 2^{-1 / 2}$ [4]. Information is therefore available for very small and very large excitations, but the previous results suggest that $\xi_{n} \sim 0.72$ for all excitations. The sign pattern follows from the fact that $\Pi_{V T}$ is ultraviolet finite and cancellations have to take place, but nothing more concrete than that can be inferred from 4-dimensional models.

The prediction from holography reads

$$
\xi_{n} \equiv \frac{f_{V n}^{\perp}}{f_{V n}}=-\pi^{2} y_{m}^{2} \kappa \rho\left(\zeta_{0, n}\right) J_{1}\left(\zeta_{0, n}\right)
$$

where $\zeta_{0, n}$ are the zeros of $J_{0}$. Interestingly, since $J_{1}\left(\zeta_{0, n}\right)$ changes sign depending on whether $n$ is odd or even, the previous expression realizes the sign pattern conjectured in [4]. Additionally, the values for $\xi_{\rho}$ and $\xi_{n>>0}$ above fix the boundary function to behave like $\rho(q)=\rho_{1} \sqrt{q y_{m}}$. 
Another quantity of interest is the magnetic susceptibility of the quark condensate, which can be defined as

$$
\lim _{q^{2} \rightarrow 0} \Pi_{V T}\left(q^{2}\right)=-\chi_{0}\langle\bar{\psi} \psi\rangle
$$

and has an impact on the determination of the hadronic light-by-light contribution to the muon $(g-2)$. Present values for $\chi_{0}$ span over the large window $2 \mathrm{GeV}^{-2} \lesssim \chi_{0} \lesssim 9 \mathrm{GeV}^{-2}$, which induces a potential $(10-15) \%$ systematic uncertainty in $(g-2)_{H L B L}[10]$. Taking the low energy limit of $\Pi_{V T}$, it is straightforward to obtain the holographic prediction:

$$
\frac{\kappa}{2} y_{m} \rho(0)=\chi_{0}\langle\bar{\psi} \psi\rangle=-\sum_{n}^{\infty} \frac{f_{V n}^{2}}{m_{V n}} \xi_{n} .
$$

Since $\rho(0) \sim 0$, it follows that our model predicts $\chi_{0} \sim 0$. In the last line above $\chi_{0}$ is split into its resonance contributions. Because of $\xi_{n}$ the different contributions will have opposite signs (and eventually cancel out to yield $\chi_{0}=0$ ). The surprising feature is that all resonances turn out to give the same contribution, and therefore lowest meson dominance fails dramatically.

\section{Conclusions}

The study of spin-1 vector mesons is not restricted to the phenomenology of vector currents. $J_{\mu}$ only describes the so-called transverse $1^{--}$mesons, while $J_{\mu v}$ generates the longitudinal $1^{--}$ mesons and, additionally, $1^{+-}$states. In the holographic language, this entails that one needs to incorporate 1-form and 2-form fields at the same time on the gravity side. The strong interrelations between them constraint both the space of allowed holographic models (the presence of dilaton backgrounds is shown to lead to inconsistencies) and the phenomenology. In this paper I have shown that a minimal model with only the kinetic term for 1-forms and 2-forms is already capable of giving a complete picture of spin-1 fields, in which chiral symmetry breaking is not introduced by hand but rather arises as a self-consistency requirement.

\section{References}

[1] J. M. Maldacena, Adv. Theor. Math. Phys. 2, 231 (1998) [Int. J. Theor. Phys. 38, 1113 (1999)] [arXiv:hep-th/9711200];

[2] J. Erlich, E. Katz, D. T. Son and M. A. Stephanov, Phys. Rev. Lett. 95, 261602 (2005) [arXiv:hep-ph/0501128].

[3] N. S. Craigie and J. Stern, Phys. Rev. D 26, 2430 (1982).

[4] O. Cata and V. Mateu, Phys. Rev. D 77, 116009 (2008) [arXiv:0801.4374 [hep-ph]].

[5] L. Cappiello, O. Cata and G. D'Ambrosio, arXiv:1004.2497 [hep-ph].

[6] E. Witten, Adv. Theor. Math. Phys. 2, 253 (1998) [arXiv:hep-th/9802150].

[7] A. Karch, E. Katz, D. T. Son and M. A. Stephanov, Phys. Rev. D 74, 015005 (2006) [arXiv:hep-ph/0602229].

[8] J. Hirn and V. Sanz, JHEP 0512, 030 (2005) [arXiv:hep-ph/0507049].

[9] D. Becirevic, V. Lubicz, F. Mescia and C. Tarantino, JHEP 0305, 007 (2003) [arXiv:hep-lat/0301020].

[10] L. Cappiello, O. Cata and G. D'Ambrosio, arXiv:1009.1161 [hep-ph]. 